PROCEEDINGS OF THE

AMERICAN MATHEMATICAL SOCIETY

Volume 130, Number 4, Pages 1065-1071

S 0002-9939(01)06315-8

Article electronically published on October 5, 2001

\title{
A NOTE ON IDEMPOTENTS IN FINITE AW*-FACTORS
}

\author{
GABRIEL NAGY \\ (Communicated by David R. Larson)
}

\begin{abstract}
We prove that the value of the quasi-trace on an idempotent element in an $\mathrm{AW}^{*}$-factor of type $\mathrm{II}_{1}$ is the same as the dimension of its left (or right) support.
\end{abstract}

It is a long-standing open problem (due to Kaplansky) to prove that an $A W^{*}$ factor of type $I I_{1}$ is in fact a von Neumann algebra. A remarkable answer, in the affirmative, was found by Haagerup ([Ha] $)$, who proved that if an $\mathrm{AW}^{*}$-factor $A$ is generated by an exact $\mathrm{C}^{*}$-algebra, then $A$ is indeed a von Neumann algebra.

The main object, that was investigated in connection with Kaplansky's problem, is the quasi-trace, whose construction we briefly recall below.

One starts with an $\mathrm{AW}^{*}$-factor of type $\mathrm{II}_{1}$, say $A$. Denote by $\mathcal{P}(A)$ the collection of projections in $A$, that is

$$
\mathcal{P}(A)=\left\{p \in A: p=p^{*}=p^{2}\right\} .
$$

A key fact is then the existence of a (unique) dimension function $D: \mathcal{P}(A) \rightarrow[0,1]$ with the following properties:

- $D(p)=D(q) \Longleftrightarrow p \sim q$;

- if $p \perp q$, then $D(p+q)=D(p)+D(q)$;

- $D(1)=1$.

The symbol " $\sim$ " denotes the Murray-von Neumann equivalence relation ( $p \sim q \Leftrightarrow$ $\exists x \in A$ with $p=x^{*} x$ and $\left.q=x x^{*}\right)$, while " $\perp$ " denotes the orthogonality relation ( $p \perp q \Leftrightarrow p q=0$; this implies that $p+q$ is again a projection).

Once the dimension function is defined, it is extended to self-adjoint elements with finite spectrum. More explicitly, if $a \in A$ is self-adjoint with finite spectrum, then there are (real) numbers $\alpha_{1}, \ldots, \alpha_{n}$ and pairwise orthogonal projections $p_{1}, \ldots, p_{n}$, such that $a=\sum_{k=1}^{n} \alpha_{k} p_{k}$. We then define $d(a)=\sum_{k=1}^{n} \alpha_{k} D\left(p_{k}\right)$.

For an arbitrary self-adjoint element $a \in A$, one can approximate uniformly $a$ with a sequence $\left(a_{n}\right)_{n \geq 1} \in\{a\}^{\prime \prime}$ of elements with finite spectrum. (Here $\{a\}^{\prime \prime}$ stands for the $\mathrm{AW}^{*}$-subalgebra generated by $a$ and 1.) It turns out that the limit $q(a)=\lim _{n \rightarrow \infty} d\left(a_{n}\right)$ is independent of the particular choice of $\left(a_{n}\right)_{n \geq 1}$.

Finally, for an arbitrary element $x \in A$, one defines $Q(x)=q(\operatorname{Re} x)+i q(\operatorname{Im} x)$, where $\operatorname{Re} x=\frac{1}{2}\left(x+x^{*}\right)$ and $\operatorname{Im} x=\frac{1}{2 i}\left(x-x^{*}\right)$.

Received by the editors October 4, 2000.

1991 Mathematics Subject Classification. Primary 46L10; Secondary 46L30.

Key words and phrases. $\mathrm{AW}^{*}$-algebra, quasi-trace, idempotent, projection, dimension function.

This work was partially supported by NSF grant DMS 9706858.

(C)2001 American Mathematical Society 
The map $Q: A \rightarrow \mathbb{C}$, defined this way, is the unique one with the properties:

(i) $Q$ is linear, when restricted to abelian $\mathrm{C}^{*}$-subalgebras of $A$;

(ii) $Q\left(x^{*} x\right)=Q\left(x x^{*}\right) \geq 0$, for all $x \in A$;

(iii) $Q(x)=Q(\operatorname{Re} x)+i Q(\operatorname{Im} x)$, for all $x \in A$;

(iv) $Q(1)=1$.

It is obvious that $\left.Q\right|_{\mathcal{P}(A)}=D$. The map $Q$ is called the quasi-trace of $A$.

It is well known that an $\mathrm{AW}^{*}$-factor of type $\mathrm{II}_{1}$ is a von Neumann algebra if and only if its quasi-trace is linear. Haagerup's solution for Kaplansky's problem goes through the proof of the linearity of the quasi-trace.

On the one hand, one can easily see that the linearity of the quasi-trace is equivalent to its scalar homogeneity (compare with (i) above):

$$
Q(\alpha x)=\alpha Q(x), \text { for all } x \in A, \alpha \in \mathbb{C} .
$$

Notice that $(\mathrm{H})$ holds when either $\alpha \in \mathbb{R}$ or when $x$ is normal. On the other hand, it is again easy to note that the linearity of the quasi-trace is equivalent to the similarity invariance property

$$
Q\left(s x s^{-1}\right)=Q(x), \text { for all } x \in A, s \in G L(A) .
$$

(Here $G L(A)$ denotes the group of invertible elements in $A$.) Notice that (S) is true if $s$ is unitary.

The purpose of this note is to prove that both $(\mathrm{H})$ and $(\mathrm{S})$ hold if $x \in A$ is an idempotent (i.e. $x^{2}=x$ ).

1. Notations. If $A$ is an $\mathrm{AW}^{*}$-algebra, for an element $x \in A$, we denote by $\mathbf{L}(x)$ (resp. $\mathbf{R}(x)$ ) the left (resp. right) support of $x$. Recall that both $\mathbf{L}(x)$ and $\mathbf{R}(x)$ are projections, and moreover we have

$$
\mathbf{L}(x) \sim \mathbf{R}(x) \text {, for all } x \in A .
$$

It is known (see $\mathrm{Ka}$ ) that, for every $x \in A$, there exists a unique partial isometry $v$ such that

(i) $x=v\left(x^{*} x\right)^{1 / 2}$;

(ii) $v v^{*}=\mathbf{L}(x)$

(iii) $v^{*} v=\mathbf{R}(x)$.

(This property is referred to as the Polar Decomposition.)

2. Remark. If $A$ is a finite $\mathrm{AW}^{*}$-algebra, then the group $G L(A)$, of invertible elements, is dense in $A$ in the norm topology.

Indeed, on the one hand, since $\mathbf{L}(x) \sim \mathbf{R}(x)$, by the finiteness assumption we also have $1-\mathbf{L}(x) \sim 1-\mathbf{R}(x)$. In particular, there exists a partial isometry $w \in A$ such that $1-\mathbf{L}(x)=w w^{*}$ and $1-\mathbf{R}(x)=w^{*} w$. On the other hand, if we take $x=v\left(x^{*} x\right)^{1 / 2}$ to be the polar decomposition described above, then we obviously have $w^{*} v=0$, so the element $u=v+w$ is unitary, and we still have $x=u\left(x^{*} x\right)^{1 / 2}$. Then, for every $\varepsilon>0$ the positive element $\left(x^{*} x\right)^{1 / 2}+\varepsilon 1$ is invertible, and so is $u\left\{\left(x^{*} x\right)^{1 / 2}+\varepsilon 1\right\}$. The result then follows from the obvious equality $\left\|x-u\left\{\left(x^{*} x\right)^{1 / 2}+\varepsilon 1\right\}\right\|=\varepsilon$.

3. Notation. For a $\mathrm{C}^{*}$-algebra $A$ and an integer $n \geq 2$, we denote by $M_{n}(A)$ the $\mathrm{C}^{*}$-algebra of $n \times n$ matrices with coefficients in $A$. 
The key technical result in this paper is the following.

4. Lemma. Suppose $A$ is a unital $C^{*}$-algebra and $x \in G L(A)$. Then there exist a unitary element $U \in M_{2}(A)$ and elements $y, z \in A$, such that

$$
U^{*}\left[\begin{array}{ll}
2 & x \\
0 & 0
\end{array}\right] U=\left[\begin{array}{ll}
1 & y \\
z & 1
\end{array}\right]
$$

Proof. Consider the function defined by

$$
f(t)=\frac{(1-t)^{2}}{t}, 0<t<1
$$

It is obvious that $f:(0,1) \rightarrow(0, \infty)$ is a homeomorphism. Since $x$ is invertible, the spectrum of $x x^{*}$ is contained in $(0, \infty)$, so by functional calculus there exists an invertible positive element $w \in A$, with $\|w\|<1$, such that $f(w)=x x^{*}$, which means

$$
x x^{*}=(1-w)^{2} w^{-1} .
$$

Define the elements

$$
\begin{array}{ll}
a=(1+w)^{-1 / 2} w^{1 / 2}, & b=(1+w)^{-1 / 2}, \\
c=x^{-1}(1+w)^{-1 / 2} w^{-1 / 2}(1-w), & d=-x^{-1}(1+w)^{-1 / 2}(1-w) .
\end{array}
$$

First, we have

$$
a a^{*}+b b^{*}=(1+w)^{-1 / 2}[w+1](1+w)^{-1 / 2}=1,
$$

and using (2) we also have

$$
\begin{aligned}
c c^{*}+d d^{*} & =x^{-1}(1+w)^{-1 / 2}(1-w)\left[w^{-1}+1\right](1-w)(1+w)^{-1 / 2}\left(x^{*}\right)^{-1} \\
& =x^{-1}(1-w)^{2} w^{-1}\left(x^{*}\right)^{-1}=x^{-1}\left(x x^{*}\right)\left(x^{*}\right)^{-1}=1 .
\end{aligned}
$$

Secondly, since by taking inverses, (2) yields

$$
\left(x^{*}\right)^{-1} x^{-1}=w(1-w)^{-2},
$$

so we also get

$$
\begin{aligned}
a^{*} a+c^{*} c & =w^{1 / 2}(1+w)^{-1 / 2}\left[1+(1-w)\left(x^{*}\right)^{-1} x^{-1}(1-w)\right](1+w)^{-1 / 2} w^{1 / 2} \\
& =w^{1 / 2}(1+w)^{-1 / 2}\left[1+w^{-1}\right](1+w)^{-1 / 2} w^{1 / 2}=1, \\
b^{*} b+d^{*} d & =(1+w)^{-1 / 2}\left[1+(1-w)\left(x^{*}\right)^{-1} x^{-1}(1-w)\right](1+w)^{-1 / 2} \\
& =(1+w)^{-1 / 2}[1+w](1+w)^{-1 / 2} 1 .
\end{aligned}
$$

Finally, we notice that

$$
a c^{*}+b d^{*}=(1+w)^{-1 / 2}\left[w^{1 / 2} w^{-1 / 2}-1\right](1-w)(1+w)^{-1 / 2}\left(x^{*}\right)^{-1}=0,
$$

and using (5) we also have

(9)

$$
\begin{aligned}
a^{*} b+c^{*} d & =(1+w)^{-1 / 2}\left[w^{1 / 2}-w^{-1 / 2}(1-w)\left(x^{*}\right)^{-1} x(1-w)\right](1+w)^{-1 / 2} \\
& =(1+w)^{-1 / 2}\left[w^{1 / 2}-w^{-1 / 2} w\right](1+w)^{-1 / 2}=0 .
\end{aligned}
$$

If we define the matrix $U=\left[\begin{array}{ll}a & b \\ c & d\end{array}\right]$, then (3), (4), and (8) give $U U^{*}=I$, while (6), (17), and (9) give $U^{*} U=I$, so $U$ is indeed unitary. (Here $I$ denotes the unit in $M_{2}(A)$.) 
Let us now observe that

$$
\begin{aligned}
2 a+x c & =2(1+w)^{-1 / 2} w^{1 / 2}+(1+w)^{1 / 2}(1-w) w^{-1 / 2} \\
& =(1+w)^{-1 / 2}\left(w^{1 / 2}+w^{-1 / 2}\right)=a+b w^{-1 / 2}, \\
2 b+x d & =2(1+w)^{-1 / 2}-(1+w)^{-1 / 2}(1-w) \\
& =(1+w)^{-1 / 2}(w+1)=a w^{1 / 2}+b, \\
c+d w^{-1 / 2} & =x^{-1}(1+w)^{-1 / 2}\left[(1-w) w^{-1 / 2}-(1-w) w^{-1 / 2}\right]=0, \\
c w^{1 / 2}+d & =\left(c+d w^{-1 / 2}\right) w^{1 / 2}=0 .
\end{aligned}
$$

These equalities prove exactly that

$$
\left[\begin{array}{ll}
2 & x \\
0 & 0
\end{array}\right] \cdot\left[\begin{array}{ll}
a & b \\
c & d
\end{array}\right]=\left[\begin{array}{cc}
1 & w^{1 / 2} \\
w^{-1 / 2} & 1
\end{array}\right] \cdot\left[\begin{array}{ll}
a & b \\
c & d
\end{array}\right]
$$

The next result is a particular case of the main result.

5. Lemma. Let $A$ be an $A W^{*}$-factor of type $I I_{1}$, and let $e \in A$ be an idempotent with $D(\mathbf{L}(e))=\frac{1}{2}$. Then, for any $\lambda \in \mathbb{C}$, we have

$$
Q(\lambda e)=\lambda / 2 .
$$

Proof. Denote, for simplicity, the projection $\mathbf{L}(x)$ by $p$. The assumption is that $p \sim 1-p$. Then we have a $*$-isomorphism $\Phi: A \rightarrow M_{2}(p A p)$, such that $\Phi(p)=$ $\left[\begin{array}{ll}1 & 0 \\ 0 & 0\end{array}\right]$. It is obvious that, since $e=p e$, there exists an element $x \in p A p$ such that $\Phi(e)=\left[\begin{array}{ll}1 & 0 \\ x & 0\end{array}\right]$. By Remark 2, we can find a sequence $\left(x_{n}\right)_{n \geq 1}$ of invertible elements in $p A p$ (the unit in $p A p$ is $p$ ) with $\lim _{n \rightarrow \infty}\left\|x_{n}-x\right\|=0$. Define the sequence

$$
E_{n}=\left[\begin{array}{cc}
1 & x_{n} \\
0 & 0
\end{array}\right] \in M_{2}(p A p), n \geq 1
$$

By Lemma 4, one can find two sequences $\left(y_{n}\right)_{n \geq 1}$ and $\left(z_{n}\right)_{n \geq 1}$ in $p A p$, and a sequence of unitaries $\left(U_{n}\right)_{n \geq 1} \subset M_{2}(p A p)$, such that

$$
E_{n}=U_{n}\left[\begin{array}{cc}
\frac{1}{2} & y_{n} \\
z_{n} & \frac{1}{2}
\end{array}\right] U_{n}^{*}, \text { for all } n \geq 1
$$

Define $e_{n}=\Phi^{-1}\left(E_{n}\right), u_{n}=\Phi^{-1}\left(U_{n}\right)$, and $a_{n}=u_{n}^{*} e_{n} u_{n}-\frac{1}{2} 1, n \geq 1$, so that we have

$$
\Phi\left(a_{n}\right)=\left[\begin{array}{cc}
0 & y_{n} \\
z_{n} & 0
\end{array}\right], \text { for all } n \geq 1
$$

Now fix a complex number $\lambda$. On the one hand, we have

$$
\lambda e_{n}=u_{n}\left(\frac{\lambda}{2}+a_{n}\right) u_{n}^{*}=\frac{\lambda}{2} 1+u_{n} a_{n} u_{n}^{*}, \text { for all } n \geq 1 .
$$

This gives, for every $n \geq 1$, the equalities

$$
\begin{aligned}
& \operatorname{Re}\left(\lambda e_{n}\right)=\left(\operatorname{Re} \frac{\lambda}{2}\right) 1+\operatorname{Re}\left(\lambda u_{n} a_{n} u_{n}^{*}\right), \\
& \operatorname{Im}\left(\lambda e_{n}\right)=\left(\operatorname{Im} \frac{\lambda}{2}\right) 1+\operatorname{Im}\left(\lambda u_{n} a_{n} u_{n}^{*}\right) .
\end{aligned}
$$


Notice however that, using the unitary invariance (property (S) for $s$ unitary), together with (17) and (18), gives, for every $n \geq 1$, the equalities

$$
\begin{aligned}
Q\left(\lambda e_{n}\right) & =Q\left(\left(\operatorname{Re} \frac{\lambda}{2}\right) 1+\operatorname{Re}\left(\lambda u_{n} a_{n} u_{n}^{*}\right)\right)+i Q\left(\left(\operatorname{Im} \frac{\lambda}{2}\right) 1+\operatorname{Im}\left(\lambda u_{n} a_{n} u_{n}^{*}\right)\right) \\
& =\operatorname{Re} \frac{\lambda}{2}+Q\left(\operatorname{Re}\left(\lambda u_{n} a_{n} u_{n}^{*}\right)\right)+i \operatorname{Im} \frac{\lambda}{2}+i Q\left(\operatorname{Im}\left(\lambda u_{n} a_{n} u_{n}^{*}\right)\right) \\
& =\frac{\lambda}{2}+Q\left(\lambda u_{n} a_{n} u_{n}^{*}\right)=\frac{\lambda}{2}+Q\left(u_{n}\left(\lambda a_{n}\right) u_{n}^{*}\right)=\frac{\lambda}{2}+Q\left(\lambda a_{n}\right) .
\end{aligned}
$$

On the other hand, if we define $v=1-2 p$ (which is obviously a unitary in $A$ ), then for all $n \geq 1$ we have

$$
\Phi\left(v\left(\lambda a_{n}\right) v^{*}\right)=\left[\begin{array}{cc}
1 & 0 \\
0 & -1
\end{array}\right] \cdot\left[\begin{array}{cc}
0 & \lambda y_{n} \\
\lambda z_{n} & 0
\end{array}\right] \cdot\left[\begin{array}{cc}
1 & 0 \\
0 & -1
\end{array}\right]=\left[\begin{array}{cc}
0 & -\lambda y_{n} \\
-\lambda z_{n} & 0
\end{array}\right]=\Phi\left(-\lambda a_{n}\right) .
$$

This gives

$$
v\left(\lambda a_{n}\right) v^{*}=-\lambda a_{n}, \text { for all } n \geq 1 .
$$

Using the unitary invariance, combined with real scalar homogeneity (property $(\mathrm{H})$ with $\alpha \in \mathbb{R}$ ), equality (20) gives

$$
Q\left(\lambda a_{n}\right)=Q\left(v\left(\lambda a_{n}\right) v^{*}\right)=Q\left(-\lambda a_{n}\right)=-Q\left(\lambda a_{n}\right),
$$

which forces

$$
Q\left(\lambda a_{n}\right)=0, \text { for all } n \geq 1 .
$$

Combining (21) with (19) gives

$$
Q\left(\lambda e_{n}\right)=\lambda / 2 \text {, for all } n \geq 1 .
$$

It is obvious that, by construction, we have $\lim _{n \rightarrow \infty}\left\|E_{n}-\Phi(e)\right\|=0$, which means that $\lim _{n \rightarrow \infty}\left\|e_{n}-e\right\|=0$. Using the norm continuity of the quasi-trace (see $[\mathrm{BH}]$ ), combined with (22), gives the desired result.

We are now ready to prove the main result.

6. Theorem. Let $A$ be an $A W^{*}$-factor of type $I I_{1}$, and let $e \in A$ be an idempotent element. Then, for any $\lambda \in \mathbb{C}$, one has the equality

$$
Q(\lambda e)=\lambda D(\mathbf{L}(e)) .
$$

Proof. The proof will be carried out in two steps.

Particular Case: Assume $D(\mathbf{L}(e)) \leq \frac{1}{2}$.

Denote, for simplicity $\mathbf{L}(e)$ by $p$, and $\mathbf{R}(e)$ by $q$. By the Paralellogram Law (see Ka] ) we have

$$
p \vee q-p \sim q-p \wedge q
$$

Since $p \sim q$, we get

$$
D(p \vee q-p)=D(q-p \wedge q)=D(q)-D(p \wedge q) \leq D(q)=D(p) .
$$

Using the intermediate value property for $D$, we can find a projection $r \leq 1-p \vee q$ such that $D(r)+D(p \vee q-p)=D(p)$. Put $q_{0}=r+p \vee q-p$. We have $p \perp q_{0}$ and $p+q_{0} \geq p \vee q$. Let us work in the AW*-algebra $A_{0}=\left(p+q_{0}\right) A\left(p+q_{0}\right)$. Obviously $A_{0}$ is again an $\mathrm{AW}^{*}$-factor of type $\mathrm{II}_{1}$, so it carries its quasi-trace $Q_{0}$. By the uniqueness of the quasi-trace, it is obvious that

$$
Q_{0}(x)=\frac{Q(x)}{D\left(p+q_{0}\right)} \text {, for all } x \in A_{0} .
$$


Notice that $e \in(p \vee q) A(p \vee q)$, so in particular $e$ belongs to $A_{0}$. In $A_{0}$, we have $\mathbf{L}(e)=p$, and $p \sim q_{0}=1_{A_{0}}-p$, which means that $D_{0}(p)=\frac{1}{2}$. (Here $D_{0}$ denotes the dimension function in $\left.A_{0}\right)$. By Lemma 5 , we get

$$
Q_{0}(\lambda e)=\lambda / 2,
$$

which combined with (24) yields

$$
\frac{\lambda}{2}=\frac{Q(\lambda e)}{D\left(p+q_{0}\right)}=\frac{Q(\lambda e)}{2 D(p)},
$$

which obviously proves (23).

General Case. One knows (see $\left[\mathrm{Be}\right.$ ) that $M_{2}(A)$ is also an $\mathrm{AW}^{*}$-factor of type $\mathrm{II}_{1}$. Moreover, if we denote by $Q^{(2)}$ the quasi-trace of $M_{2}(A)$, we have (as above)

$$
Q(x)=2 Q^{(2)}\left(\left[\begin{array}{ll}
x & 0 \\
0 & 0
\end{array}\right]\right) \text {, for all } x \in A .
$$

Define the idempotent $E=\left[\begin{array}{ll}e & 0 \\ 0 & 0\end{array}\right] \in M_{2}(A)$, and the projection $P=\left[\begin{array}{ll}p & 0 \\ 0 & 0\end{array}\right] \in M_{2}(A)$. Using (25) we have $Q(e)=2 Q^{(2)}(E)$. If we denote by $D^{(2)}$ the dimension function of $M_{2}(A)$, then by (25) we also have $D(p)=2 D^{(2)}(P)$, which gives $D^{(2)}(P) \leq \frac{1}{2}$. Using the obvious equality $\mathbf{L}(E)=P$, by the particular case above applied to $M_{2}(A)$, we get

$$
Q(\lambda e)=2 Q^{(2)}(\lambda E)=2 \lambda D^{(2)}(P)=\lambda D(p) .
$$

7. Corollary. Let $A$ be an $A W^{*}$-factor of type $I I_{1}$, and let $e \in A$ be an idempotent. Then, for every $s \in G L(A)$, we have

$$
Q\left(\operatorname{ses}^{-1}\right)=Q(e) .
$$

Proof. Put $p=\mathbf{L}(e)$. If we define $t=1-e(1-p)$, then $t$ is invertible, and $e=t p t^{-1}$. This computation shows that it is enough to prove (26) in the case when $e=e^{*}$. Let $q=\mathbf{L}\left(\operatorname{ses}^{-1}\right)$. Arguing as above, there exists some $x \in G L(A)$ such that $s e s^{-1}=x q x^{-1}$. So if we put $y=x^{-1} s$, we have $y e y^{-1}=q$, with both $e$ and $q$ self-adjoint idempotents. Since this obviously forces $e \sim q$, we get $D(e)=D(q)=Q\left(\right.$ ses $\left.^{-1}\right)$.

8. Corollary. Let $A$ be an $A W^{*}$-factor of type $I_{1}$ and let $e_{1}, e_{2} \in A$ be idempotents, such that $e_{1} e_{2}=e_{2} e_{1}=0$. (This implies that $e_{1}+e_{2}$ is again an idempotent.) Then

$$
Q\left(e_{1}+e_{2}\right)=Q\left(e_{1}\right)+Q\left(e_{2}\right) .
$$

Proof. Let $p=\mathbf{L}\left(e_{1}+e_{2}\right)$. As in the proof of the preceding corollary, there exists an element $s \in G L(A)$, such that $p=s\left(e_{1}+e_{2}\right) s^{-1}$. Put $f_{k}=s e_{k} s^{-1}, k=1$, 2, so that $f_{1}+f_{2}=p$. But now we also have $p f_{k}=f_{k} p=f_{k}, k=1,2$, which means in particular that $f_{1}, f_{2} \in p A p$. So, if we work in the $\mathrm{AW}^{*}$-factor (again of type $\mathrm{II}_{1}$ ) $A_{0}=p A p$, we will have $f_{1}+f_{2}=1_{A_{0}}$. On the one hand, using the notations from the proof of Theorem 6 , we have

$$
Q_{0}\left(f_{1}\right)+Q_{0}\left(f_{2}\right)=1 .
$$

On the other hand, we have

$$
Q_{0}\left(f_{k}\right)=\frac{Q\left(f_{k}\right)}{D(p)}, \quad k=1,2
$$


so we get

$$
D(p)=Q\left(f_{1}\right)+Q\left(f_{2}\right) .
$$

Finally, using the preceding corollary, we get

$$
Q\left(e_{1}+e_{2}\right)=D(p)=Q\left(f_{1}\right)+Q\left(f_{2}\right)=Q\left(s e_{1} s^{-1}\right)+Q\left(s e_{2} s^{-1}\right)=Q\left(e_{1}\right)+Q\left(e_{2}\right) .
$$

9. Comment. A. Theorem 6 can be analyzed from a different point of view, as follows. In principle, one can extend the dimension function $D$ to the collection of all idempotents by $\tilde{D}(e)=D(\mathbf{L}(e))$. One can easily prove that this extended dimension function will have the same properties as the usual dimension function (when Murray-von Neumann equivalence is extended to idempotents). The point of Theorem 6 is then the fact that $\tilde{D}=Q$.

B. It is interesting to note that the linearity of the quasi-trace is equivalent to the following condition:

(*) For any family of idempotents $e_{1}, \ldots, e_{n} \in A$, such that $e_{j} e_{k}=0$ for all $j, k \in\{1, \ldots, n\}$ with $j \neq k$, and any family of numbers $\alpha_{1}, \ldots, \alpha_{n} \in \mathbb{R}$, one has:

$$
Q\left(\alpha_{1} e_{1}+\cdots+\alpha_{n} e_{n}\right)=\alpha_{1} Q\left(e_{1}\right)+\cdots+\alpha_{n} Q\left(e_{n}\right) .
$$

This will be discussed in a future paper.

\section{REFERENCES}

[Be] S. Berberian, Baer *-rings, Springer, 1972 MR 46:7294

[BH] B. Blackadar, D. Handelman, Dimension functions and traces on $C^{*}$-algebras, J. Funct. Anal. 45 (1982), no. 3, 297-340. MR 83g:46050

[Ha] U. Haagerup, Quasi-traces on exact $\bar{C}^{*}$-algebras are traces, Preprint, 1991

[Ka] I. Kaplansky, Rings of operators, Benjamin, 1968 MR 39:6092

Department of Mathematics, Kansas State University, Manhattan, Kansas 66506

E-mail address: nagy@math.ksu.edu 